140s

\title{
1. Antidepressant effect of tianeptine: the paradox between clinical and neurochemical data
}

EUROPEAN MULTICENTRE STUDY OF TIANEPTINE VERSUS IMIPRAMINE AND PLACEBO IN THE TREATMENT OF MAJOR DEPRESSION AND DEPRESSED BIPOLAR DISORDER.

L. Staner* A. Bertolino, G.B. Cassano. J. De Wilde. L. Ferreira. A. Kasas, J.Mendlewicz, C. Mertens. M. Paes de Sousa. P. Sarteschi. H.A. Van. H. Lôo

* Service de Psychiatrie, Centre Hospitalier, Luxembourg

186 depressed patients were included in a multicentre study comparing tianeptine with imipramine and placebo. Diagnoses were : Major Depression, Bipolar Disorder (depressed). They were treated with tianeptine ( $37.5 \mathrm{mg} /$ day) or imipramine $(150 \mathrm{mg} /$ day) or placebo for 42 days. Efficacy assessments were : MÅDRS, CGI, HARS, HSCL and a VAS. 57 patients dropped-out (placebo $n=23$, tianeptine $n=17$, imipramine $n=17$ ). Reasons for discontinuation differed for placebo and imipramine groups : more inefficacy on placebo, more side-effects on imipramine. In patients treated for at least 14 days $(n=171)$, final MÅDRS scores showed a better efficacy of tianeptine and imipramine than placebo. In patients treated for 42 days $(n=129)$ the time courses of MÁDRS scores were significantly different $(p=0.036)$ with final scores of $16.1,11.8$ and 14.3 on placebo, tianeptine and imipramine respectively (62\% decrease in MADRS score on tianeptine, $54 \%$ on imipramine and $48 \%$ on placebo). Responders in all patients $(n=186)$ were $32 \%$ on placebo, $56 \%$ on tianeptine and $48 \%$ on imipramine, with a better efficacy of tianeptine when compared to placebo. CGI scale (item 1) showed a better efficacy of tianeptine and imipramine than placebo in the whole study population. HARS showed a better efficacy of tianeptine and imipramine than placebo for psychic anxiety. Dry mouth, constipation, hot flushes were more frequent on imipramine than on tianeptine or placebo. These results support the efficacy of tianeptine $(37.5 \mathrm{mg} /$ day $)$ in the treatment of Major Depression and Depressed Bipolar Disorder when compared to placebo. No difference was found between tianeptine and imipramine (150 mg/day) for efficacy and between tianeptine and placebo for safety. TIANEPTINE VERSUS PLACEBO STUDY IN MAJOR
DEPRESSIONS AND DEPRESSIVE BIPOLAR DISORDERS

\section{LA, COSTA E SILVA,S. RUSCHEL}

Hospital Santa Casa da Misericordia - Rio de Janeiro, Brazil

\begin{abstract}
A multicentre study aiming to compare the efficacy and safety of tianeptine with those of placebo in the treatment of Major Depressions and Depressed Bipolar Disorders was performed in Brazil.

126 depressed patients ( $78 \%$ females, $22 \%$ males) were included in this trial. They presented either a Major Depression, single episode (38\%), or a Major Depression, recurrent $(60 \%)$ or a Bipolar Disorder, depressed (2\%). $60 \%$ of the patients fulfilled DSMIII R criteria for melancholia. They were treated with either tianeptine ( $37.5 \mathrm{mg} /$ day) or placebo for 42 days, after a 7-day run-in placebo period. Efficacy assessments were MÅDRS, CGI, HARS, Zung Scale and a VAS. Safety assessments included physical examinations, laboratory parameters and AMDPS scale.

20 patients discontinued the treatment (placebo $n=9$, tianeptine $\mathrm{n}=11$ ). Final MADRS scores in the intention to treat analysis showed a better efficacy of tianeptine than placebo $(p=0.007)$; this was confirmed in the patients treated for at least 14 days $(p=0.008)$, and in the patients treated for 42 days $(p=0.027)$. CGI item 1 (severity of illness) confirmed the better efficacy of tianeptine. Responders $\geq 50 \%$ decrease in MÅDRS initial scores) were $41 \%$ on placebo and $58 \%$ on tianeptine. The overall efficacy judgement of the investigators confirmed $(p=0.027)$ the efficacy of tianeptine with $62 \%$ of patients improved on tianeptine versus $40 \%$ on placebo. Furthermore, there was a trend for a better efficacy of taneptine versus placebo on HARS psychic anxiety subscore. Safety was satisfactory for both compounds. Mild adverse events were observed on tianeptine (headache, constipation).

This study shows the efficacy of tianeptine in the treatment of Major Depressions and Depressed Bipolar Disorders with or without Melancholia in a placebo controlled study.
\end{abstract}


ELECTROPHYSIOLOGICAL EFFECTS OF ACUTE AND LONG-TERM TIANEPTINE ADMINISTRATION ON SEROTONIN NEUROTRANSMISSION

P Blier, G Piñevro, L Deveault. C de Montigny

Neurobiological Psychiatry Unit, Department of Psychiatry, McGill

University, 1033 Pine avenue west, H3A 1A1, Montréal, Canada.

The tricyclic drug tianeptine has previously been shown to increase $e x$ vivo the uptake of $\left[{ }^{3} \mathrm{H}\right] 5-\mathrm{HT}$. In the present study, acute intravenous injection and microiontophoretic application of tianeptine were found to increase the firing activity of dorsal hippocampus $\mathrm{CA}_{3}$ pyramidal neurons in chloral hydrate anesthetized rats. The enhancing effect of local application of tianeptine was unaltered in rats with lesioned 5-HT neurons, indicating that it is not mediated by an increased effectiveness of the 5-HT transporter. Nevertheless, long-term (20 $\mathrm{mg} / \mathrm{kg} /$ day for 14 days, delivered by an osmotic minipump implanted subcutaneously), but not acute ( $2 \mathrm{mg} / \mathrm{kg}$, intravenously), tianeptine did reduce the time for $\mathrm{CA}_{3}$ neurons to recover their firing activity following microiontophoretic application of 5-HT. Consistent with these results, the effectiveness of the selective 5-HT reuptake blocker paroxetine to prolong the suppression of firing produced by microiontophoretically-applied 5-HT was decreased after 14 days of treatment with tianeptine, but not after its acute administration. This effect of long-term tianeptine was no longer present after a 48-hour washout. We then assessed whether long-term tianeptine altered overall 5-HT neurotransmission with the minipump in place. Treatments of two, seven and 14 days altered neither the firing rate of dorsal raphe 5-HT neurons, nor the sensitivity of their somatodendritic $5-\mathrm{HT}_{1 \mathrm{~A}}$ autoreceptors. The effectiveness of the electrical stimulation of the 5-HT pathway in suppressing the firing activity of hippocampus $\mathrm{CA}_{3}$ neurons was also unaltered in long-term tianeptine treated rats. These electrophysiological results obtained in vivo indicate that, whereas acute tianeptine administration does not modify the 5-HT reuptake process, a long-term treatment can alter the 5-HT transporter as long as tianeptine is present in the brain. This, however, does not result in a detectable alteration of 5-HT neurotransmission in the rat hippocampus.
EFFECT OF A SINGLE DOSE OF TIANEPTINE IN HEALTHY VOLUNTEER ON SLEEP ELECTROPHYSIOLOGICAL PARAMETERS

\section{STANER* J, MENDLEWICZ** \\ * Service de Psychiatrie, Centre Hospitalier, Luxembourg \\ **Hopital Universitaire Erasme - 1070 Bruxelles, Belgique}

Tianeptine is an antidepressant which may be distinguished from classical antidepressants by its neurochemical action of increasing 5-HT uptake in the synaptic cleft. Pharmacological studies have shown that acute administration of tianeptine in the rat increases active wakefulness in the first hour. Tianeptine affects neither rapid eye mouvement sleep nor slow wave sleep in the rat, in contrast to the usual action of antidepressant drugs (1). Furthermore, a similar effect has been observed after chronic administration in the monkey (2)

A specific sleep study aiming to evaluate the activity of tianeptine on sleep EEG parameters in 10 young healthy volunteers $(5 \mathrm{~F}, 5 \mathrm{M}$; mean age : $31.6 \pm 6.0$ ) has been set up. In this study, after an adaptation night, three nights were used for the assessment of sleep EEG parameters: a first night (N1) for Baseline, and 2 nights (N2, N3) for the comparison of the effects of tianeptine and placebo.

Night N1 was on placebo, and nights N2 and N3 on tianeptine and placebo, in a cross over design. Tianeptine was given at the dose of

$12.5 \mathrm{mg}$ and the time of administration was $10 \mathrm{pm}$. The main sleep EEG parameters were : sleep onset latency, number and total duration of awakenings, total duration of stages $1,2,3$, and 4, REM sleep onset latency, total duration of REM sleep. Furthermore the sleep questionnaire of Leeds was used in order to record the subjects impressions on their sleep quality.

(1) Lejeune F., Poignant J.C., Reure H. - Etude électrophysiologique de la tianeptine, nouveau stimulant du recaptage de la sérotonine possédant une activité antidépressive. - Neurophysiol. Clin., 1988, 18, 369-381

(2) Mocaër E., Lagarde D., Balzamo E., Milhaud C. - Effects of tianeptine on sleep-wakefulness cycles and EEG in monkeys. Psychopharmacology, 1988, 96 (suppl), 31.02.33., P275 